\title{
A Terahertz Grid Frequency Doubler
}

\author{
Alina Moussessian, Member, IEEE, Michael C. Wanke, Yongjun Li, Jung-Chih Chiao, S. James Allen, \\ Thomas W. Crowe, Senior Member, IEEE, and David B. Rutledge, Fellow, IEEE
}

\begin{abstract}
We present a 144-element terahertz quasi-optical grid frequency doubler. The grid is a planar structure with bowtie antennas as a unit cell, each loaded with a planar Schottky diode. The maximum output power measured for this grid is $24 \mathrm{~mW}$ at $1 \mathrm{THz}$ for 3.1- $\mu \mathrm{s}$ 500-GHz input pulses with a peak input power of $47 \mathrm{~W}$. An efficiency of $0.17 \%$ for an input power of $6.3 \mathrm{~W}$ and output power of $10.8 \mathrm{~mW}$ is measured. To date, this is the largest recorded output power for a multiplier at terahertz frequencies. Input and output tuning curves are presented and an output pattern is measured and compared to theory.
\end{abstract}

Index Terms-Frequency doubler, multiplier grid, quasi-optics, terahertz.

\section{INTRODUCTION}

$\mathbf{T}$ HERE IS increasing demand for terahertz components such as tunable oscillators, mixers, and multipliers for use in submillimeter-wave sources. These sources are used in radio astronomy and remote sensing [1], [2]. Conventional sources such as lasers and vacuum tubes are large and heavy. They need high-voltage power supplies and have limited tuning range. However, solid-state diode multipliers like Schottky diode multipliers can be used to generate higher harmonics from low-frequency tunable signal sources such as Gunn-diode oscillators. Currently, most of these diode multipliers are a single diode or a cascade of two or more diodes mounted in waveguide with a whisker contact. Rydberg et al. have demonstrated a Schottky varactor-diode frequency tripler with an output power more than $120 \mu \mathrm{W}$ at $803 \mathrm{GHz}$ [3]. Erickson and Tuovinen have presented a waveguide tripler with an output power of $110 \mu \mathrm{W}$ at $800 \mathrm{GHz}$ [4]. Zimmermann et al. have demonstrated a cascade of a two-whisker-contacted Schottky-varactor frequency tripler with an output power of $60 \mu \mathrm{W}$ at $1 \mathrm{THz}$ [5].

Another approach is to combine the output powers of many solid-state devices in free space, thereby eliminating the losses associated with waveguide structures. The first terahertz grid

Manuscript received March 20, 1998; revised July 27, 1998. This work was supported by the Physical Optics Corporation, by the U.S. Army Research Office, and by the Jet Propulsion Laboratory.

A. Moussessian is with the Jet Propulsion Laboratory, Pasadena, CA 91109 USA.

M. C. Wanke and S. J. Allen are with the Department of Physics, University of California at Santa Barbara, Santa Barbara, CA 93106 USA.

Y. Li was with the Department of Electrical Engineering, University of Virginia, Charlottesville, VA 22903 USA. He is now with Pricewaterhouse Coopers Securities, McLean, VA 22102 USA.

J.-C. Chiao is with the Department of Electrical Engineering, University of Hawaii at Manoa, Manoa, Honolulu, HI 96822 USA.

T. W. Crowe is with the Department of Electrical Engineering, University of Virginia, Charlottesville, VA 22903 USA.

D. B. Rutledge is with the Department of Electrical Engineering, California Institute of Technology, Pasadena, CA 91125 USA.

Publisher Item Identifier S 0018-9480(98)08338-0.

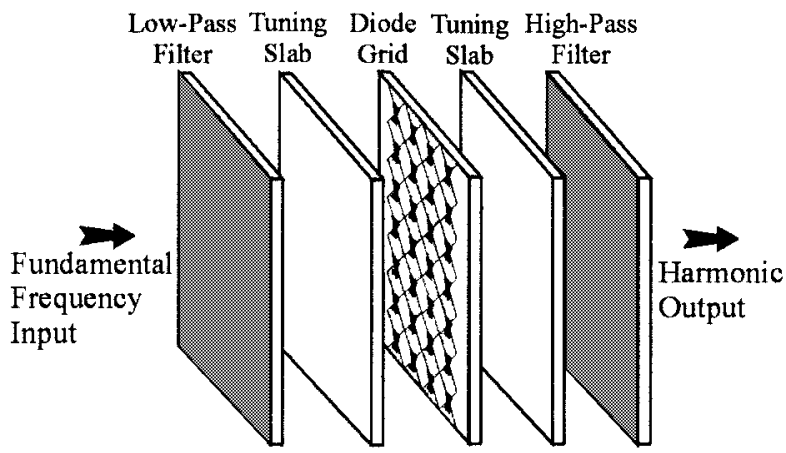

Fig. 1. A grid frequency multiplier [8]. The fundamental frequency enters the grid through a low-pass filter. The grid acts as a nonlinear surface and generates harmonics which pass through a high-pass filter.

frequency doubler was presented by Chiao et al. This $6 \times 6$ doubler grid had a peak output power of $330 \mu \mathrm{W}$ at $1 \mathrm{THz}$ [6]. This grid had a null in the output beam because of the diode orientations used. Later, a second terahertz grid doubler was presented, which corrected the diode orientation problem. The output power reported for this $12 \times 12$ grid was $5.5 \mathrm{~mW}$ at $1 \mathrm{THz}$ for 3.1- $\mu \mathrm{s} 500-\mathrm{GHz}$ input pulses with a peak power of $36 \mathrm{~W}$ [7]. In the grid presented in this paper, input and output tuners are used to increase the output power of the grid in [7]. A diode-grid frequency doubler is an array of closely spaced planar Schottky diodes placed in a setup, as shown in Fig. 1. The fundamental beam excites RF currents on the leads of the bow-ties. The diodes act as a nonlinear surface and generate harmonics. The low-pass filter in the input insures that only the fundamental frequency of the laser will hit the grid. The high-pass output filter allows the higher harmonics generated by the grid to pass through, but blocks the fundamental. The input and output tuning slabs are for impedance matching of the input and output.

\section{CONSTRUCTION}

The grid multipliers were fabricated at the University of Virginia, Charlottesville, using monolithic technology [9]. To make diodes for terahertz frequencies, series resistance and shunt junction capacitance should be greatly reduced by reducing anode diameter and choosing optimum active layer doping and thickness. Fig. 2 shows the top view and cross section of the Schottky diode. The anode has a diameter of $0.5 \mu \mathrm{m}$. A surface channel is etched under the anode contact finger to reduce the shunt capacitance. The diodes have an estimated junction capacitance of $0.6 \mathrm{fF}$ at zero bias, and a dc-series resistance of $14 \Omega$.

The grid consists of an array of $12 \times 12$ bow-tie antennas on a $30-\mu \mathrm{m}$-thick fused-quartz substrate. Fig. 3 shows the 


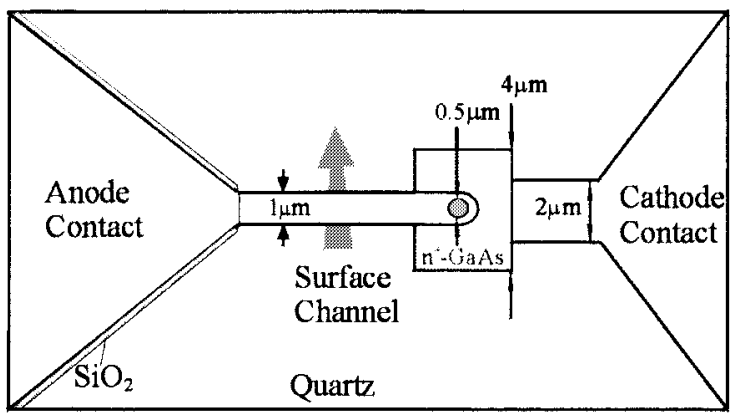

(a)

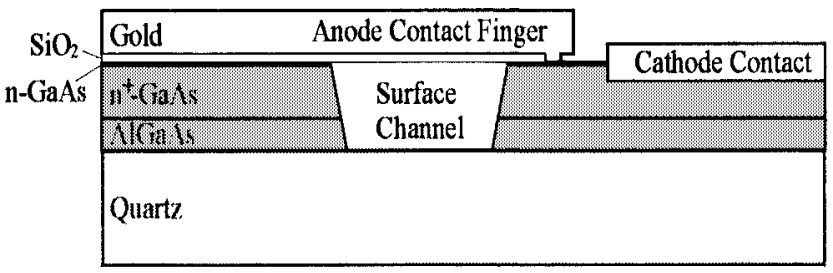

(b)

Fig. 2. Schottky-diode layout. (a) Top view. (b) Cross section. The n-GaAs layer is $0.1-\mu \mathrm{m}$ thick and has a doping concentration of $4 \times 10^{17} \mathrm{~cm}^{-3}$. The $\mathrm{n}^{+}$-GaAs layer has a thickness of $3 \mu \mathrm{m}$ and a doping of $5 \times 10^{18} \mathrm{~cm}^{-3}$. The AlGaAs layer has a thickness of $1.5 \mu \mathrm{m}$.

grid and unit cell. Each unit cell is $70 \mu \mathrm{m}$ on a side. The Schottky-diode junction is located at the center of the cell. The diode grid is first fabricated on a GaAs substrate. After the fabrication, the GaAs substrate is etched away and the diode structure is glued on a $30-\mu \mathrm{m}$-thick quartz substrate.

\section{MEASUREMENTS}

The measurement setup is shown in Fig. 4. The input source for these measurements is the free-electron laser (FEL) from the University of California at Santa Barbara [10]. The FEL is capable of generating kilowatts of pulsed power tunable from $120 \mathrm{GHz}$ to $4.8 \mathrm{THz}$. The pulsewidth in the measurements is $3.1 \mu \mathrm{s}$. The input power passes through a low-pass multimesh filter with a cutoff frequency of $660 \mathrm{GHz}$ and is varied by rotating a polarizer. The second nonrotating polarizer placed after the first one is to maintain the polarization of the beam. A beam splitter directs part of the input power into a pyroelectric reference detector. The rest of the input is focused onto the grid. A metallic-mesh Fabry-Perot interferometer is used to measure the frequency content of the output. The output beam is focused onto a liquid-helium-cooled InSb bolometer through a high-pass filter with a cutoff frequency of $870 \mathrm{GHz}$.

The diode grid is suspended in air by gluing it over a hole in a microscope cover slip. The grid is placed in the setup shown in Fig. 4 and excited by a 500-GHz input beam. There are no water-absorption lines in $500 \mathrm{GHz}$ and $1 \mathrm{THz}$ [11], therefore, measurements can be done in free space without the aid of a dry-box. The reference and output signals are synchronized with the FEL trigger signal that has a frequency of $0.75 \mathrm{~Hz}$. To measure the peak input and output power, each pulse is integrated over certain time intervals. To account for the noise in our measurements, the pulses are also integrated over the same time period before the arrival of the FEL trigger signal.

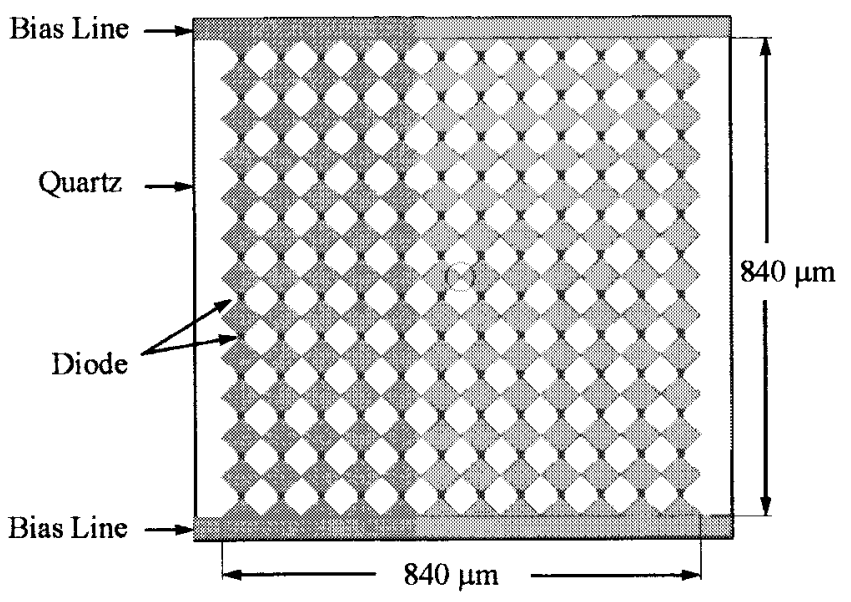

(a)

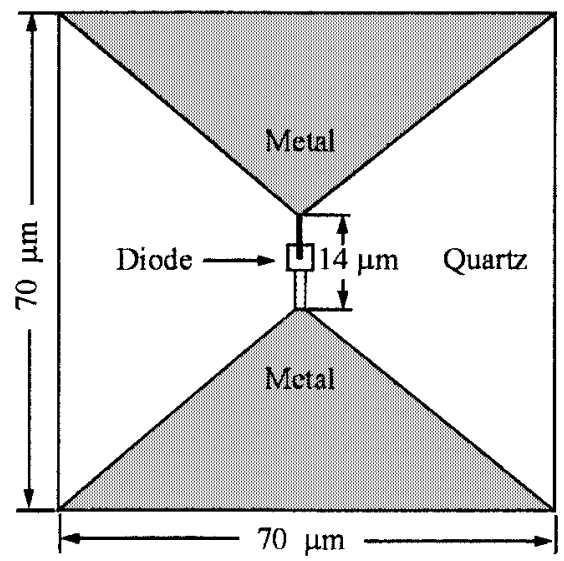

(b)

Fig. 3. The multiplier grid. (a) Entire $12 \times 12$ array. The diodes are in series from top to bottom. The grid can be biased through the bias lines at the top and bottom of the grid. The metal contacts in a row are all electrically connected. (b) Unit cell. The diode is located at the center of the cell.

This offset is subtracted from the measured peak power. This method is used for all the measurements.

To investigate whether the grid generates terahertz second harmonics, a Fabry-Perot interferometer, as shown in Fig. 4, is used to verify the output frequency of the grid. Measuring the output power versus the metal-mesh spacing of the interferometer indicates that for an input frequency of 500 $\mathrm{GHz}$, a 1-THz output signal is present. No other harmonics were detected. The grid is also tested with an input of 645 GHz. A similar Fabry-Perot measurement indicates an output at $1.29 \mathrm{THz}$. To ensure that the output radiation is actually from the grid, we removed the grid in both measurements. The output pulse disappeared. We also rotated the grid by $90^{\circ}$, which had the same effect. This shows that the output signal is not a harmonic of the laser or generated by the GaAs epitaxial layer [7].

\section{INPUT POWER MEASUREMENT}

To determine the amount of the input power incident on the grid, a pyroelectric detector mounted on a two-dimensional positioner is used to measure the spot size of the incident beam at the focal point where the grid is placed. The measured $E$ - 


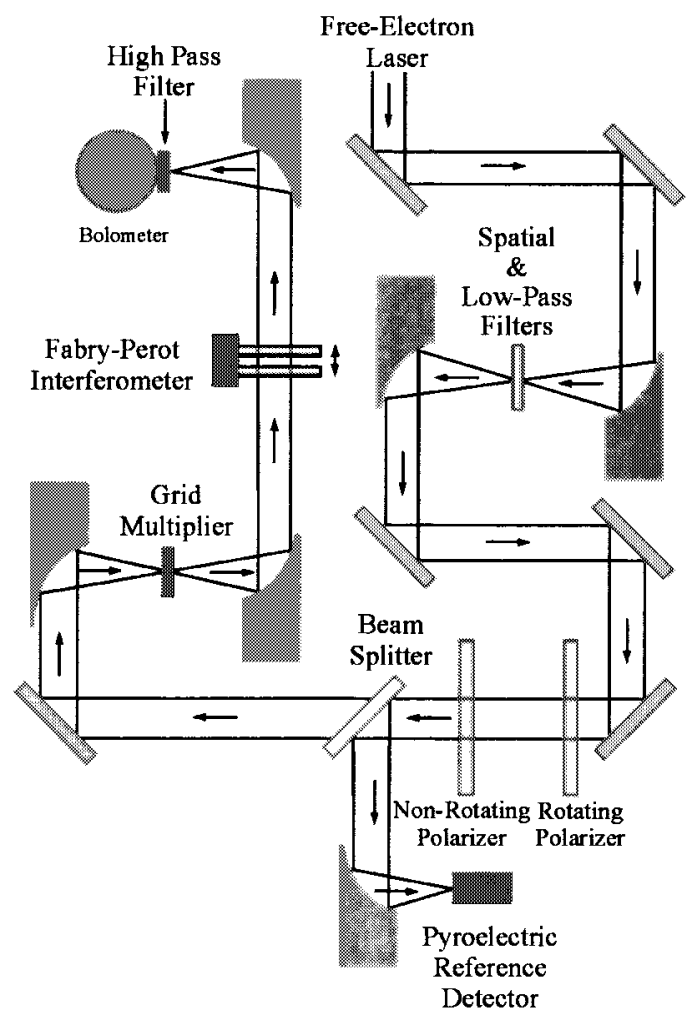

Fig. 4. Measurement setup. All the mirrors in the setup are $\mathrm{f} / 1.2$ parabolic, with focal length of $13 \mathrm{~cm}$ and diameter of $10 \mathrm{~cm}$. Arrows indicate the direction of the beam.

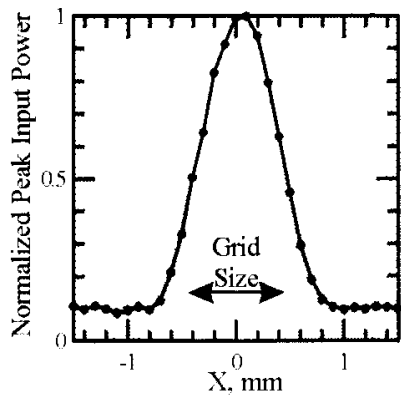

(a)

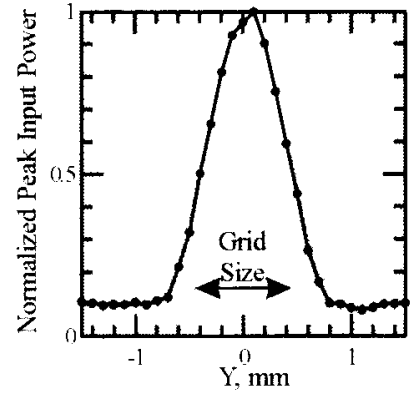

(b)
Fig. 5. Input spot-size measurement at the focal plane using a pyroelectric detector. (a) $E$-plane pattern. (b) $H$-plane pattern.

and $H$-plane patterns of a 500-GHz incident beam are shown in Fig. 5(a) and (b), respectively. Comparing the spot size of the incident beam to the grid size, which is $840 \mu \mathrm{m}$ on the side, indicates that almost all of the input power is incident on the grid.

\section{Tuning MeAsurement}

Dielectric slab tuners, as shown in Fig. 1, can be used to achieve higher output power and efficiency. We used z-cut crystal-quartz discs with a dielectric constant of 3.78 as tuners. The input tuner has a thickness of $60 \mu \mathrm{m}$ and the output tuner is $30-\mu \mathrm{m}$ thick. To investigate whether the tuners improve the power performance of the grid, we first measured the output versus the input power of the grid with no tuners (see Fig. 6).

To measure the input tuning curve, shown in Fig. 7(a), the output tuner in Fig. 1 is removed and the output power is

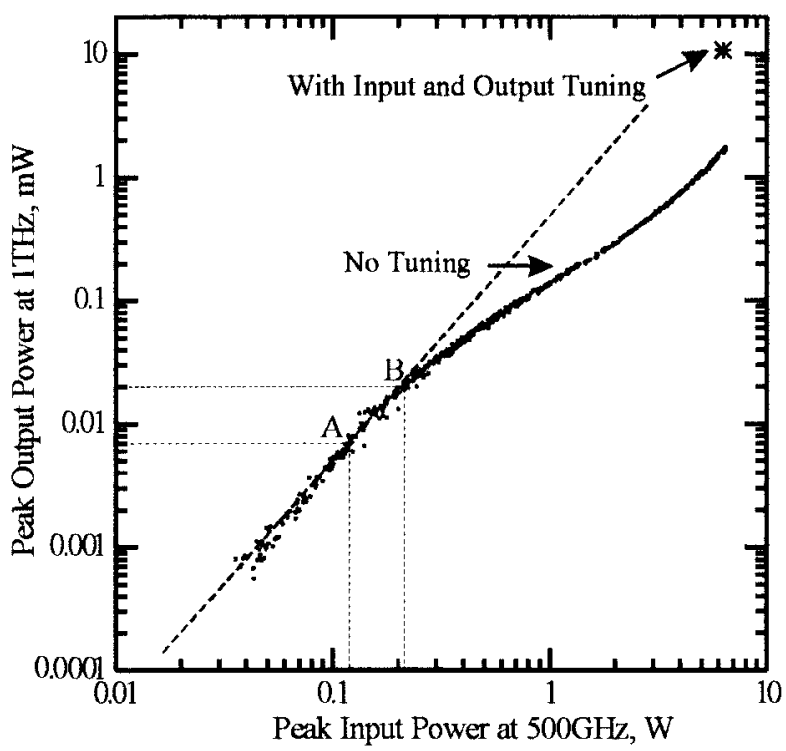

Fig. 6. Measured peak output power at $1 \mathrm{THz}$ versus the input power at $500 \mathrm{GHz}$ with and without tuners. $A$ and $B$ show the points where the input and output tuning measurements are done. The dashed line shows the square-law relationship.

measured versus the input tuner's relative distance from the grid. At its closest point to the grid [0 in Fig. 7(a)], the tuner is less than $500 \mu \mathrm{m}$ away from the grid. The average spacing between the peaks in this figure is $300 \mu \mathrm{m}$, which is a halfwavelength of the fundamental frequency. After the first two periods, there is a significant drop in the output power, which is probably due to diffraction. Point $A$ in Fig. 6 shows the input and output power of the grid for the input tuning measurement before the tuning slab is added. This corresponds to an output power of $7 \mu \mathrm{W}$, which is shown as a dashed line in Fig. 7(a). By adding the input tuner, the $7-\mu \mathrm{W}$ output power increases to $37 \mu \mathrm{W}$ [see Fig. 7(a)].

The output tuning curve is shown in Fig. 7(b). For this measurement, the input tuning slab (shown in Fig. 1) is removed and the output is measured versus the relative position of the output tuning slab from the grid. Here also, the closest distance from the grid [0 in Fig. 7(b)] is less than $500 \mu \mathrm{m}$. The average spacing between the peaks is $150 \mu \mathrm{m}$, which is half the second harmonic wavelength. Point $B$ in Fig. 6 shows the input and output powers before adding the output tuning slab. For this measurement, the output power of the grid with no tuners is $20 \mu \mathrm{W}$, shown as a dashed line in Fig. 7(b). The output tuner increases the output power from 20 to $32.5 \mu \mathrm{W}$. It appears that this tuning slab also tunes the input as is evident from the alternating high-low peaks in Fig. 7(b).

Next, with both the input and the output tuners in place (see Fig. 1), we optimized the position of these tuners for maximum output power. For an input power of $6.3 \mathrm{~W}$, an output power of $10.8 \mathrm{~mW}$ was measured, indicating an efficiency of $0.17 \%$ (see Fig. 6).

\section{MAXIMUM OUTPUT POWER}

To measure the maximum output power of the grid at $1 \mathrm{THz}$, we placed both the input and output tuners in the setup of Fig. 1, optimized their position for maximum output, and 


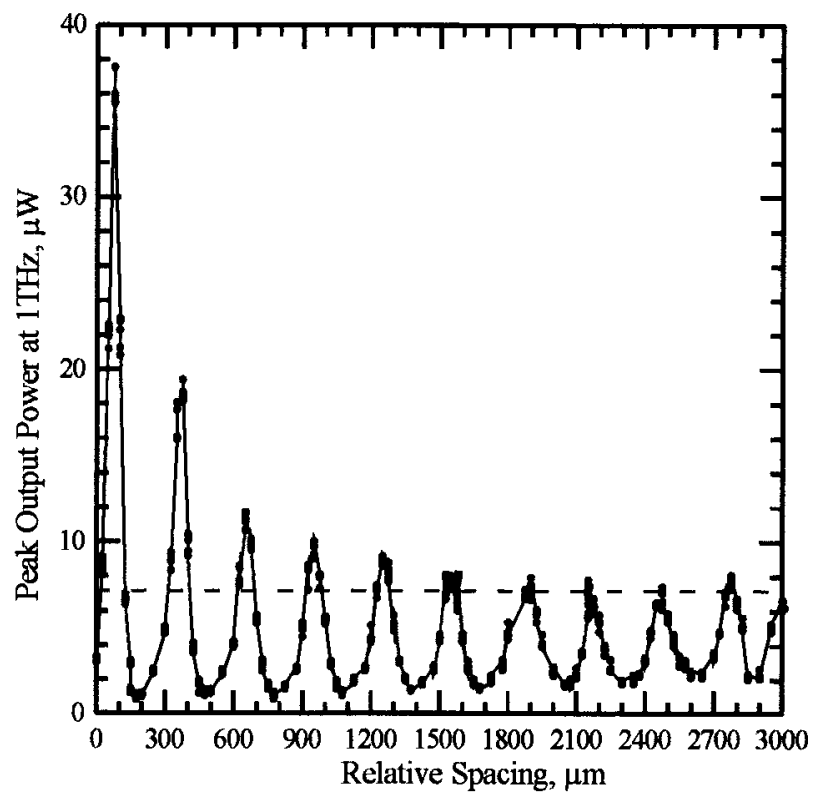

(a)

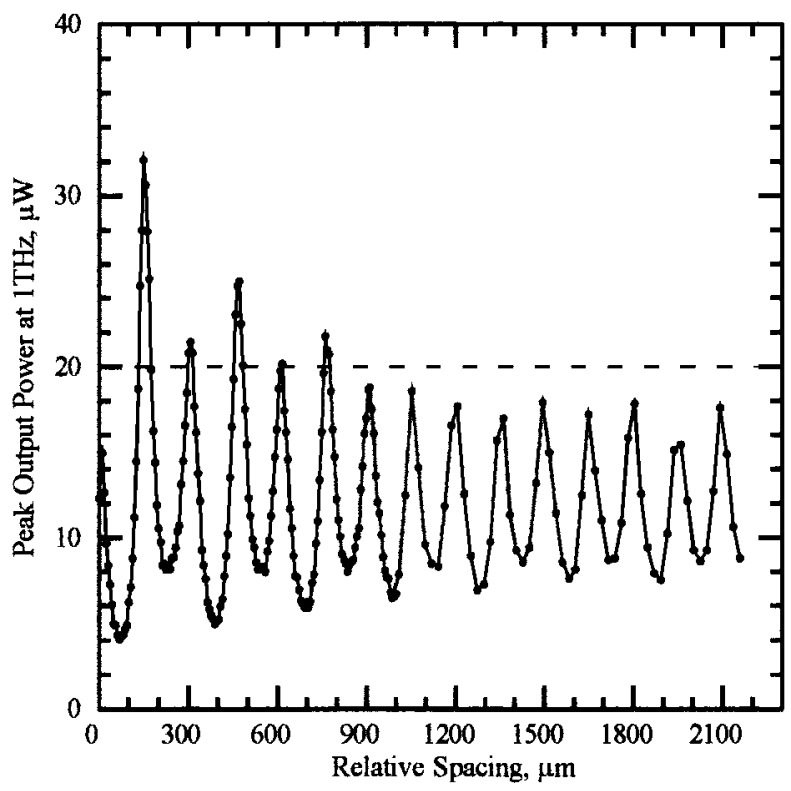

(b)

Fig. 7. Measured output power versus the relative position of (a) the input tuner and (b) output tuner. The dashed line is the output power with no tuner for each measurement.

increased the input power until the grid was damaged. Fig. 8 shows the output versus the input power measurement before the input and output tuners are added, and compares it to the same measurement with the tuners optimized for maximum output. The tuners are at a distance less than $500 \mu \mathrm{m}$ from the grid. A maximum output power of $24 \mathrm{~mW}$ is measured at $1 \mathrm{THz}$ with an input power of $47 \mathrm{~W}$ for 3.1- $\mu$ s input pulses.

At an input power of $47 \mathrm{~W}$, the output starts rolling over indicating damage to some devices. As the input power increases further, the output power starts increasing again until some more devices are damaged. Fig. 8 shows a second decrease in the output power. We stopped the measurement at this point.

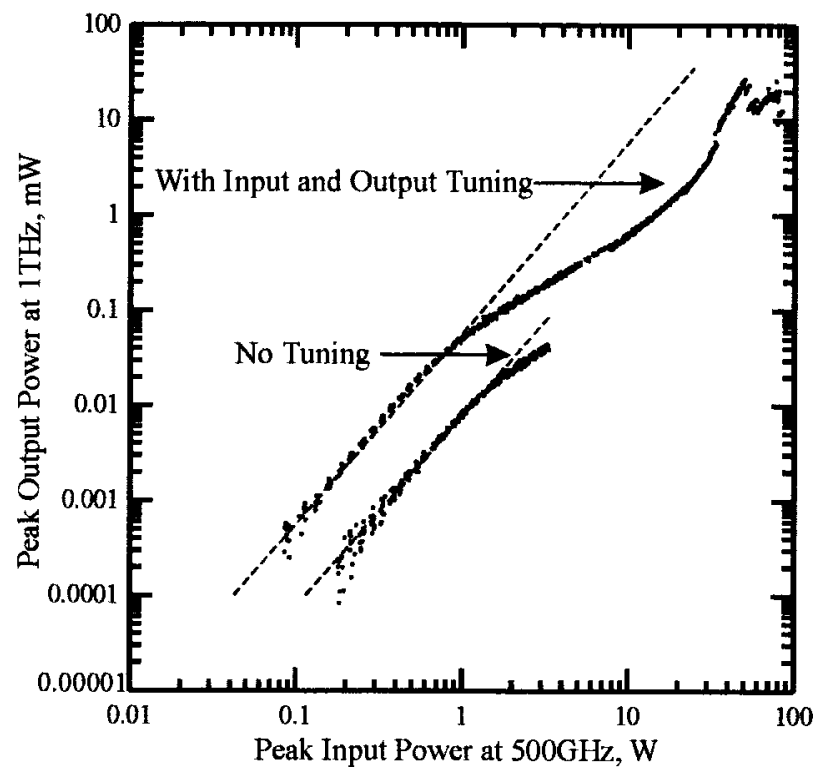

Fig. 8. Measured peak output power at $1 \mathrm{THz}$ versus input power at $500 \mathrm{GHz}$ with and without tuning. The dashed lines show the square-law relationship.

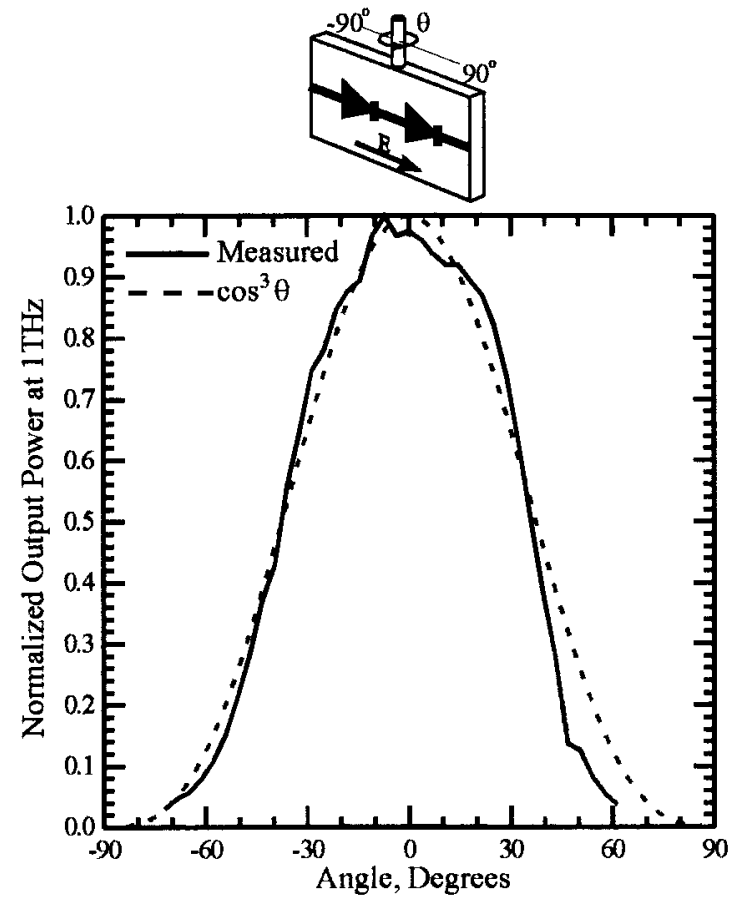

Fig. 9. Output pattern. The measurement was done by rotating the grid from $-90^{\circ}$ to $90^{\circ}$ with the electric field parallel to the diodes.

The discrepancy between the power dependence of the grid in Figs. 6 and 8 is possibly due to alignment of the grid with the input beam. With low input power, the data in both Figs. 6 and 8 follow a square-law relationship. At higher input power, there is a kink in the data for both measurements. It is possible that this kink is a self-biasing effect, where the RF power changes with the diode impedance.

\section{PATTERN MEASUREMENT}

Fig. 9 shows the effect of rotating the grid about its axis from $-90^{\circ}$ to $90^{\circ}$. Unlike the pattern for the previous doubler 
grid reported by Chiao [6], this pattern has no nulls. In the case of the previous grid, the diodes were oriented with opposite polarity about the centerline of the grid, causing a null in the output. Here, the diode orientation is corrected, eliminating the null in the pattern. The output power stays within $30 \%$ of the maximum for angles up to $30^{\circ}$. A theoretical $\cos ^{3} \theta$ obliquity factor is also plotted in this figure. Since the incident beam to the grid is approximately the same size as the grid, rotating the grid about its axis causes the input power density to reduce by a factor of $\cos \theta$. The second factor of $\cos \theta$ in this obliquity factor is because the grid is a square-law device, and the third factor is because the output beam becomes wider as the grid rotates, missing the output detector.

\section{CONCLUSION}

In this paper, we have presented a planar grid of 144 Schottky diodes suitable for use as a quasi-optical frequency doubler. This grid has a maximum efficiency of $0.17 \%$ for an output power of $10.8 \mathrm{~mW}$, and a peak output power of $24 \mathrm{~mW}$ at $1 \mathrm{THz}$ for $3.1-\mu \mathrm{s} 500-\mathrm{GHz}$ input pulses with a peak power of $47 \mathrm{~W}$.

\section{REFERENCES}

[1] T. G. Phillips, "Development in submillimeter astronomy," in 19th Int. Conf. Infrared Millimeter Waves, Sendai, Japan, Oct. 1994, pp. $141-142$.

[2] J. W. Waters and P. H. Siegel, "Applications of millimeter and submillimeter technology to earth's upper atmosphere: Results to date and potential for the future," presented at the 4th Int. Symp. Space Terahertz Technol., Los Angeles, CA, Mar. 1993.

[3] A. Rydberg, B. N. Lyons, and S. U. Lidholm, "On the development of a high efficiency $750-\mathrm{GHz}$ frequency tripler for $\mathrm{THz}$ heterodyne systems," IEEE Trans. Microwave Theory Tech., vol. 40, pp. 827-830, May 1992.

[4] N. Erickson and J. Tuovinen, "A waveguide tripler for 720-880 GHz," in 6th Int. Symp. Space Terahertz Technol., Pasadena, CA, Mar. 1995, pp. 191-198.

[5] R. Zimmermann, T. Rose, T. Crowe, and T. Grein, "An all solidstate $1 \mathrm{THz}$ radiometer for space applications," in 6th Int. Symp. Space Terahertz Technol., Pasadena, CA, Mar. 1995, pp. 13-27.

[6] J.-C. Chiao, A. Markelz, Y. Li, J. Hacker, T. Crowe, J. Allen, and D. Rutledge, "Terahertz grid frequency doublers," in 6th Int. Symp. Space Terahertz Technol., Pasadena, CA, Mar. 1995, pp. 199-206.

[7] A. Moussessian, M. C. Wanke, Y. Li, J.-C. Chiao, F. A. Hegmann, S. A. Allen, T. W. Crowe, and D. B. Rutledge, "A terahertz grid frequency doubler," in IEEE MTT-S Int. Microwave Symp., vol. 2, Denver, CO, June 1997, pp. 683-686.

[8] C. F. Jou, W. W. Lam, H. Z Chen, K. S. Stolt, N. C. Luhmann, Jr, and D. B. Rutledge, "Millimeter wave diode grid frequency doubler," IEEE Trans. Microwave Theory Tech., vol. 36, pp. 1507-1514, Nov. 1988.

[9] T. W. Crowe, R. J. Mattauch, H. P. Röser, W. L. Bishop, W. C. B. Peatman, and X. Liu, "GaAs Schottky diodes for $\mathrm{THz}$ mixing applications," Proc. IEEE, vol. 80, pp. 1827-1841, Nov. 1992.

[10] S. J. Allen, K. Craig, B. Galdrikian, J. N. Heyman, J. P. Kaminski, K. Campman, P. F. Hopkins, A. C. Gossard, D. H. Chow, M. Lui, and T. K. Liu, "Materials science in the far-IR with electrostatic-based FEL's," presented at FEL'94, Stanford, CA, Aug. 1994.

[11] H. M. Pickett, R. L. Poynter, E. A. Cohen, M. L. Delitsky, J. C. Pearson, and H. S. P. Müller, "Submillimeter, millimeter, and microwave spectral line catalogue," JPL Pub. 80-23, Rev. 4, NASA, Jet Propulsion Lab., California Inst. Technol., Pasadena, CA, Mar. 10, 1990

Alina Moussessian (S'97-M'98), for photograph and biography, see this issue, p. 1963.

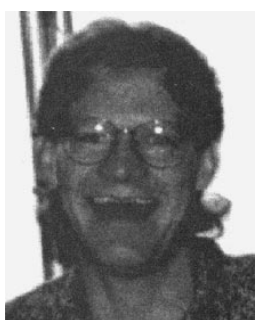

Michael C. Wanke received the B.A. degree in physics and mathematics (cum laude) from Pomona College, Claremont, CA, in 1991, the M.A. degree in physics from the University of California at Santa Barbara in 1994, and is currently working toward the Ph.D. degree.

His work experience has included the Aerospace Corporation and Lawrence Berkeley Laboratories. $\mathrm{He}$ has authored nine publications and has given four invited presentations. He is interested in terahertz device physics, and is currently exploring harmonic generation from Bloch oscillating superlattices in quasi-optical arrays. He invented a method of laser rapid prototyping of photonic bandgap microstructures.

Mr. Wanke's honors include the QUEST Vice Chancellors Fellowship (1997-1998), Dupont de Nemours Fellowship and Department of Education Graduate Fellowship (1992-1993), Frank Parkhurst Brackett Jr. and Davida Wark Brackett Prize in Astronomy (1992), Pomona College Scholar (1990, 1991), and the Achievement Reward for College Scientists (1989, 1990).

Yongjun $\mathbf{L i}$ received the B.S. degree from Beijing University, Beijing, China, the M.S. degree in electrical engineering from the University of Virginia, Charlottesville, and the M.B.A. degree from the Wharton School of Business, University of Pennsylvania, Philadelphia.

He was a Research Scientist at the University of Virginia, where he designed and fabricated high-frequency semiconductor devices. In 1998, he joined Pricewaterhouse Coopers Securities, McLean, VA, where he is currently a Senior Associate in the Technology Industry Group.

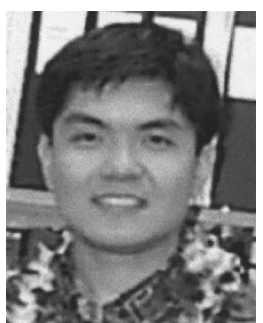

Jung-Chih Chiao received the Ph.D. degree from the California Institute of Technology, Pasadena, in 1995.

From October 1995 to August 1997, he was a Research Scientist in the Optical Networking Systems and Testbeds Group, Bell Communications Research (Bellcore), where he was involved in integrating different wavelength-division-multiplexing (WDM) network elements in the testbed of the multiwavelength optical network (MONET) consortium. He is currently an Assistant Professor in the Department of Electrical Engineering, University of Hawaii at Manoa. His research interests include monolithic microwave/millimeter-wave integrated circuits, quasi-optical millimeter-wave/submillimeter-wave components, microelectromechanical system (MEMS) RF and optical devices for wireless/high-speed systems as well as multiwavelength WDM optical networks, and WDM reconfigurable network elements and network architectures.

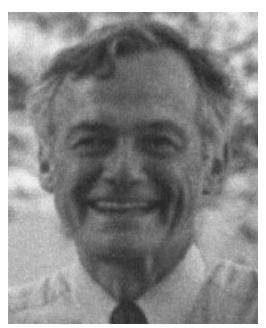

S. James Allen received the Ph.D. degree in electrical engineering from the Massachusetts Institute of Technology, Cambridge, in 1965.

Prior to his appointment at the University of California at Santa Barbara, in 1991, as Professor of physics and Director of the Quantum Institute, he was a Member of Technical Staff at Bell Laboratories, where he was recognized as a Distinguished Member of Technical Staff and availed himself of a Bell Laboratories supported leave at the Royal School of Mines, Imperial College, London, U.K He later joined Bellcore, as a District Research Manager of solid-state physics research. He has also been an Adjunct Professor at Northeastern and Princeton Universities. His current research focuses on terahertz dynamics in semiconductor quantum structures far from equilibrium, phase coherent transport in superconductor-semiconductor heterostructures and magnetoelectronics in novel magnetic semimetal-semiconductor heterostructures. $\mathrm{He}$ is currently on the editorial board of Physical Review.

Dr. Allen was elected a member at large of the Executive Committee of the Division of Condensed Matter Physics of the American Physical Society, where he served on the Strategic Planning and Nominating Committees. He is a Fellow of the American Physical Society. In 1995, he won a Humboldt Senior Science Award. 


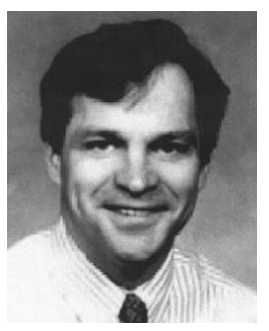

Thomas W. Crowe (S'82-M'82-SM'91) received the B.S. degree in physics from Montclair State College, Montclair, NJ, in 1980, and the M.S.E.E. degree and Ph.D. degree in electrical engineering from the University of Virginia, Charlottesville, in 1982 and 1986, respectively.

He remained at the University of Virginia, where, in March 1986, he became a Research Assistant Professor of electrical engineering, a Research Associate Professor in July 1991, and a Research Professor in August 1997. Since January 1989, he has been the Director of the Semiconductor Device Laboratory, and, in 1996, he was elected Director of the Applied Electrophysics Laboratories. He is also a founder of Virginia Diodes, Inc. His main areas of interest include the development of high-frequency semiconductor devices and the optimization of such devices for use in low-noise submillimeter-wavelength receivers. His current research is focused on the investigation of novel device structures for high-frequency applications, development of solid-state power sources for terahertz frequencies and the use of planar-device technologies to allow the routine implementation of heterodyne receivers on space platforms for radio astronomy and studies of the chemistry of the upper atmosphere.

David B. Rutledge (S'77-M'77-SM'89-F'93), for biography, see this issue, p. 1955. 